\title{
O TRABALHO EM SERVIÇOS DA REDE DE ATENÇÃO PSICOSSOCIAL: DIFICULDADES ENFRENTADAS PELOS TRABALHADORES*
}

\author{
Sonia Regina da Costa Lapischies ${ }^{1}$, Zélma Gulart De Lima², Vanda Maria da Rosa Jardim³,
} Valéria Cristina Christello Coimbra ${ }^{4}$, Luciane Prado Kantorski ${ }^{5}$

RESUMO: Para identificar os trabalhadores que enfrentam situações, as quais os incomodam no trabalho, conforme variáveis sociodemográficas e de inserção no trabalho, para descrevê-las e relacioná-las à estrutura e ao processo nos serviços de saúde mental comunitária foi realizado um estudo transversal, em 2009, por meio de questionário autoaplicado com 209 trabalhadores da região sul do Brasil. Os dados mostraram que $68,9 \%$ desses trabalhadores enfrentam situações que os incomodam no trabalho, este prevalecendo entre os trabalhadores com graduação e pós-graduação (77,5\%), com tempo de trabalho igual ou superior a 61 meses $(84,4 \%)$ e com vínculos trabalhistas não precários $(76,4 \%)$. O processo nos serviços de saúde mental comunitária concentrou a maioria das situações referidas pelos trabalhadores em causar descontentamento, tais como as relações constituídas no trabalho em equipe e os tensionamentos das relações com gestores e demais serviços da rede de saúde.

PALAVRAS-CHAVE: Saúde mental; Serviços de saúde mental; Trabalho.

\section{WORK IN SERVICES OF THE PSYCHOSOCIAL CARE NETWORK: DIFFICULTIES FACED BY THE WORKERS}

ABSTRACT: This cross-sectional study was undertaken in 2009 through a self-administered questionnaire given to 209 workers in the Southern region of Brazil. It aimed to identify the workers who face situations which disturb them at work according to socio-demographic variables and their insertion in the job, and to describe and relate them to the structure and process of the community mental health services. The data showed that $68.9 \%$ of these workers face situations which disturb them at work, with greater prevalence among workers with undergraduate or post-graduate degrees $(77.5 \%)$, with length of service equal or superior to 61 months (84.4\%) and with a secure position in their employment (76.4\%). The majority of the situations mentioned by the workers as causing discontent were concentrated in the community mental health services' process, examples being the relationships which form in team work and tension in the relationships with managers and other services in the health network. KEYWORDS: Mental health; Mental health services; Work.

\section{EL TRABAJO EN SERVICIOS DE LA RED DE ATENCIÓN PSICOSOCIAL: DIFICULTADES AFRONTADAS POR LOS TRABAJADORES}

RESUMEN: Para identificar los trabajadores que afrontan situaciones, las cuales los molestan en el trabajo, de acuerdo con variables sociodemográficas y de inserción en el trabajo, para describirlas y relacionarlas a la estructura y al proceso en los servicios de salud mental comunitaria, se realizó un estudio transversal, en 2009, por medio de cuestionario autoaplicado con 209 trabajadores de la región sur de Brasil. Los datos mostraron que 68,9\% de eses trabajadores afrontan situaciones que los molestan en el trabajo. Eso prevalece entre trabajadores con graduación y posgrado (77,5\%), con tiempo de trabajo igual o superior a 61 meses $(84,4 \%$ y con vínculos de trabajo no precarios $(76,4 \%)$. El proceso en los servicios de salud mental comunitaria concentró la mayoría de las situaciones referidas por los trabajadores en causar descontentamiento, tales como las relaciones constituidas en el trabajo en equipo y las tensiones de las relaciones con gestores y demás servicios de la red de salud.

PALABRAS CLAVE: Salud mental;Servicios de salud mental; Trabajo.

* Artigo apresentado à disciplina Redação de Artigo Científico, do Programa de Pós-Graduação - Mestrado em Enfermagem da Faculdade de Enfermagem da Universidade Federal de Pelotas - PPGENF UFPel.

${ }^{1}$ Enfermeira. Mestranda pelo PPGENF UFPel. Membro do Grupo de Pesquisas em Enfermagem, Saúde Mental e Saúde Coletiva.

${ }^{2}$ Enfermeira. Especialista em Saúde da Família e em Saúde Mental. Mestranda pelo PPGENF UFPel. Membro do Grupo de Pesquisas em Enfermagem, Saúde Mental e Saúde Coletiva.

${ }^{3}$ Enfermeira. Doutora em Enfermagem. Professora da Faculdade de Enfermagem UFPel. Membro do Grupo de Pesquisas em Enfermagem, Saúde Mental e Saúde Coletiva.

${ }^{4}$ Enfermeira. Doutora em Enfermagem em Saúde Pública.Professora da Faculdade de Enfermagem da UFPel. Membro do Grupo de Pesquisas em Enfermagem, Saúde Mental e Saúde Coletiva.

${ }^{5}$ Enfermeira. Doutora em Enfermagem. Professora da Faculdade de Enfermagem UFPel. Líder do Grupo de Pesquisas em Enfermagem, Saúde Mental e Saúde Coletiva .

\section{Autor correspondente:}

Sonia Regina da Costa Lapischies

Recebido: 18/08/2012

Universidade Federal de Pelotas

Rua Almirante Barroso, 1056 - 96010-280 - Pelotas-RS-Brasil

E-mail: sonia_lapisx@hotmail.com 


\section{INTRODUÇÃO}

O movimento pela Reforma Psiquiátrica no Brasil eclodiu na década de 1970 juntamente ao movimento sanitário que buscava mudanças nos modelos de gestão e práticas de atenção nos serviços de saúde. Trabalhadores em saúde mental que integravam o movimento sanitário uniram-se às associações de familiares, sindicalistas, membros de associações de profissionais e pessoas com longo histórico de internações psiquiátricas e deram início ao movimento social pelos direitos dos pacientes psiquiátricos em nosso país. A partir desse movimento foi construída, coletivamente, uma crítica ao saber psiquiátrico vigente e ao modelo hospitalocêntrico no cuidado às pessoas em sofrimento psíquico ${ }^{(1)}$.

Atualmente, a Política de Saúde Mental no Brasil apresenta-se sustentada na Lei 10.216/02 que busca a consolidação de um modelo de atenção à saúde mental aberto, de base comunitária. Este modelo conta com uma rede de serviços e equipamentos variados e, entre estes, podemos citar os Centros de Atenção Psicossocial (CAPS), os Serviços Residenciais Terapêuticos (SRT), os Centros de Convivência e Cultura e os leitos de atenção integral (em Hospitais Gerais, nos CAPS III) ${ }^{(2)}$.

Este estudo está focado em dois serviços, CAPS e SRT, e mais especificamente, em seus trabalhadores. Os CAPS são definidos como dispositivos que buscam promover o cuidado dos usuários em conjunto com as suas famílias e a comunidade, estimulando sua integração social e familiar, apoiando suas iniciativas de busca da autonomia e oferecendo atendimento multidisciplinar. Os SRT são residências terapêuticas, ou simplesmente moradias, localizadas no espaço urbano. Essas têm a finalidade de responder à necessidade de moradia de pessoas com transtornos mentais, egressas de longos períodos de internação em hospitais psiquiátricos, hospitais de custódia ou em situação de vulnerabilidade ${ }^{(3)}$.

$\mathrm{O}$ cuidado em liberdade às pessoas em sofrimento psíquico investe nas potencialidades de articulação das redes de apoio ${ }^{(1)}$ e, para seu sucesso, o saber técnico de cada profissão é extrapolado e construído um saber plural e integrado de equipe. A interdisciplinariedade é uma necessidade decorrente do fenômeno complexo e multifacetado do adoecer psíquico ${ }^{(4)}$.

As equipes dos serviços que operam no modo psicossocial utilizam grande variedade de instrumentos de trabalho, desde os conhecimentos especializados de cada área, técnicas em grupos terapêuticos, oficinas culturais, artísticas e de geração de renda, constituindo-se em recursos e agentes do processo de trabalho ${ }^{(4)}$.
Conhecer as percepções dos trabalhadores sobre seu trabalho contribui para a compreensão da dinâmica dos serviços inseridos no modo psicossocial.

Diante do exposto, o presente artigo tem por objetivos: identificar os trabalhadores de SRT e CAPS que enfrentam situações que incomodam em seu trabalho conforme variáveis sociodemográficas e de inserção no trabalho, e descrever as referidas situações, relacionando-as à estrutura e ao processo nos serviços de saúde mental comunitária.

\section{MÉTODO}

Este artigo apresenta um recorte descritivo do estudo 'Redes que reabilitam: avaliando experiências inovadoras de composição de redes de atenção psicossocial' - REDESUL, edital MCT-CNPq/CT-Saúde/ MS-SCTIE-DECIT/ 33-2008 e utiliza dados de questionário autoaplicado a 209 trabalhadores que representavam a integralidade dos funcionários lotados nos serviços, excetuando aqueles em férias ou licença. $\mathrm{O}$ estudo foi realizado em 39 serviços (6 CAPS e 33 SRT) de cinco municípios do Rio Grande do Sul (Alegrete, Bagé, Caxias do Sul, Porto Alegre e Viamão), serviços esses definidos a partir da existência de SRT no município, característica considerada como marcador para existência de rede de atenção psicossocial ${ }^{(5)}$.

A coleta de dados ocorreu entre setembro e dezembro de 2009 e foi antecedida por oficinas de sensibilização e estudo-piloto a fim de testar instrumentos, fazer adaptações necessárias e selecionar entrevistadores. O controle de qualidade foi realizado por meio de de checagem na entrega do questionário, revisão dos supervisores ao receber o questionário, replicação de $10 \%$ dos questionários, correção da codificação, dupla digitação e limpeza dos dados por digitadores independentes.

O projeto de pesquisa foi aprovado pelo Comitê de Ética em Pesquisa da Faculdade de Odontologia da Universidade Federal de Pelotas, sob o parecer de número 073/2009 de 14 de janeiro de 2009, financiado pelo MCT-CNPq/CT-Saúde/MS-SCTIE-DECIT/33/2008. O estudo está em concordância com os preceitos éticos exigidos para pesquisa com seres humanos, atendendo o constante na Resolução 196/96 do Conselho Nacional de Saúde.

Para coleta de dados foi utilizado instrumento constituído de 56 questões, abertas e fechadas, e a definição do desfecho deste estudo foi feita a partir da pergunta: Em seu trabalho o senhor(a) enfrenta situações que o(a) incomodam? Se sim, quais as situações que o(a) incomodam? A partir de resposta positiva ao primeiro questionamento, os trabalhadores tinham possibilidade de elencar até quatro 
situações, que foram codificadas posteriormente.

As variáveis independentes estudadas foram: sexo; idade (17-30 anos, 31-45 anos e 46-65 anos); serviço (CAPS ou SRT); escolaridade (fundamental, médio, técnico e graduação/pós-graduação); tempo de trabalho (0-12 meses, 13-60 meses e 61-450 meses) e vínculo empregatício (considerado não precário para estatutário e celetista e precário para os demais vínculos).

A partir das situações que incomodam no trabalho, referidas pelos trabalhadores realizou-se agrupamento, conforme a característica da situação elencada. Utilizando-se o referencial de Donabedian ${ }^{(6)}$, dois grupos foram constituídos: de estrutura e de processo.

A análise foi realizada por meio das frequências das situações que incomodam no trabalho e análise bivariada dos trabalhadores que enfrentam as situações com variáveis sociodemográficas e variáveis de inserção nos serviços utilizando-se o teste qui-quadrado, ep-valor menor que 0,05 .

\section{RESULTADOS}

Dentre o total de situações referidas pelos trabalhadores ( $\mathrm{n}=244)$, a grande concentração de situações que incomodam no trabalho (74\%) está relacionada ao processo e as demais (26\%) à estrutura.

Os dados da tabela 1 descrevem as situações referidas pelos trabalhadores agrupadas em estrutura e processo. Entre as situações que incomodam o trabalhador na estrutura, a falta de recursos humanos foi referida em $25 \%$ das respostas, seguida pela estrutura física $(17,18 \%)$ que compreende estrutura física inadequada, espaço físico limitado e condições físicas inadequadas, e recursos financeiros $(15,62 \%)$, abrangendo falta de recursos financeiros e não repasse de verba ao CAPS.

No processo, a situação referida com maior frequência em incomodar o trabalhador no trabalho foi dificuldade e/ou problema na equipe (42,5\%), compreendendo falta de comprometimento com pacientes e com a equipe, principalmente em situações de crise, falta de cooperação intraequipe, conflitos por melhor divisão das atribuições e responsabilidades e deterioração das relações de trabalho. A segunda situação mais referida foi caracterizada por gestão (18,33\%), abrangendo demora da gestão no reparo das inadequações, não valorização do trabalhador pela Secretaria Municipal de Saúde, falta de apoio das esferas Estadual e Federal, falta de apoio da rede de Atenção à Saúde, falta de supervisão, difícil suporte da secretaria da saúde, demanda reprimida por falta de vagas para usuários nos serviços, pressões externas para ingresso de novos usuários e tráfico de influências. Esta situação foi seguida por rede de atenção e serviço (12,5\%), abarcando problemas no acompanhamento dos usuários após a saída do SRT, dificuldades de integração à rede geral de saúde e atenção em Saúde Mental não funciona como rede.

Tabela 1 - Distribuição das situações que incomodam os trabalhadores de Centros de Atenção Psicossocial e Serviços Residenciais Terapêuticos no trabalho conforme estrutura e processo. Rio Grande do Sul, 2009

\begin{tabular}{lcc}
\hline VARIÁVEIS & N & $\mathbf{\%}$ \\
\hline Estrutura & $\mathbf{6 4}$ & $\mathbf{1 0 0}$ \\
Recursos Humanos & 16 & 25 \\
Estrutura Física & 11 & 17,18 \\
Recursos Financeiros & 10 & 15,62 \\
Materiais & 9 & 14,06 \\
Segurança & 8 & 12,5 \\
Equipamentos & 5 & 7,81 \\
Condições Insalubres & 3 & 4,68 \\
Medicamentos & 2 & 3,12 \\
Processo & $\mathbf{1 8 0}$ & $\mathbf{1 0 0}$ \\
Dificuldade/Problema Equipe & 77 & 42,77 \\
Gestão & 33 & 18,33 \\
Rede de Atenção e Serviço & 23 & 12,77 \\
Atenção a Crise & 17 & 9,44 \\
Sobrecarga da Equipe & 15 & 8,33 \\
Comunidade & 8 & 4,44 \\
Atenção Psicossocial & 7 & 3,88 \\
\hline
\end{tabular}

De um total de 209 trabalhadores de SRT/CAPS que participaram do estudo, 144 (68,9\%) responderam positivamente quando perguntados se enfrentam situações que os incomodam no trabalho. Avaliada a prevalência do desfecho estudado, enfrentar situações que incomodam no trabalho, de acordo com as variáveis sexo, idade e escolaridade, não houve diferença significativa entre os sexos e entre as faixas etárias, que apresentaram $\mathrm{p}$ valor $>0,05$, apesar de ter ocorrido crescimento na prevalência a cada grupo de idade. Contudo a prevalência em enfrentar situações que incomodam no trabalho de acordo com a escolaridade foi de $77,5 \%$ entre trabalhadores com curso de graduação e/ou pós-graduação, significativamente superior aos trabalhadores com escolaridade até ensino técnico ( $p$ valor $=0,003$ ), como apresentado na tabela 2 .

Quando analisamos as variáveis relacionadas à inserção do trabalhador no serviço identificamos associações com tempo de trabalho e com vínculo empregatício. Os trabalhadores lotados no serviço por período maior do que 60 meses são os que mais en- 
frentam situações que incomodam no trabalho $(84,4 \%)$, seguidos por aqueles que estão no serviço por período de 13 a 60 meses $(69,1 \%)$.

Avaliado o vínculo trabalhista, os trabalhadores com vínculo considerado não precário (celetistas e estatutários) foram os que mais referiram enfrentar situações que incomodam no trabalho $(76,4 \%) \mathrm{em}$ relação àqueles com vínculo precário. Não foi identificada associação estatisticamente significativa entre tipo de serviço (SRT ou CAPS) no qual o trabalhador está inserido e ao enfrentar situações que incomodam no trabalho $(\mathrm{p}$ valor $=0,55)$.

Tabela 2 - Prevalência de situações que incomodam trabalhadores de Centros de Atenção Psicossocial e Serviços Residenciais Terapêuticos, conforme variáveis sociodemográficas e de inserção no serviço. Rio Grande do Sul, 2009

\begin{tabular}{lccc}
\hline VARIÁVEL & $\mathbf{N}$ & $\mathbf{\%}$ & $\mathbf{p}$-valor \\
\hline Sexo (n=209) & & & $\mathbf{0 , 1 5}$ \\
Masculino & 53 & 62,3 & \\
Feminino & 156 & 71,2 & \\
Idade em anos (n=209) & & & $\mathbf{0 , 2 8}$ \\
17-30 & 47 & 59,6 & \\
$31-45$ & 83 & 71,1 & \\
46-65 & 79 & 72,2 & \\
Escolaridade (n=209) & & & $\mathbf{0 , 0 0 3}$ \\
Fundamental/médio/técnico & 98 & 59,2 & \\
Graduação/Pós-Graduação & 111 & 77,5 & \\
Serviço & & & $\mathbf{0 , 5 5}$ \\
SRT & 103 & 68,9 & \\
CAPS & 106 & 68,9 & \\
Tempo de trabalho (n=207) & & & $\mathbf{0 , 0 2}$ \\
0-12 meses & 65 & 60,0 & \\
13-60 meses & 97 & 69,1 & \\
61-450 meses & 45 & 84,4 & \\
Vínculo (n=208) & & & $\mathbf{0 , 0 0 0 3}$ \\
Não precário & 144 & 76,4 & \\
Precário & 64 & 51,6 & \\
\hline
\end{tabular}

\section{DISCUSSÃO}

É possível que o tamanho da amostra seja pequeno para evidenciar diferenças estatisticamente significativas além das identificadas. Particularmente a variável idade mostrou tendência de aumento na prevalência do desfecho com o aumento da idade em cada extrato sem, contudo, apresentar diferença estatística significativa.

Ao analisarmos as situações referidas pelos trabalhadores, no componente estrutura, a falta de recursos humanos, item citado maior número de vezes, pode estar refletindo a não disponibilidade de uma equipe multiprofissional para atender as necessidades de cuidado no modo psicossocial. O trabalho em equipe é um diferencial na atividade em saúde mental, e direcionado ao cuidado aos usuários e seus familiares, a fim de reduzir, progressivamente, as internações e capacitar a integração à sociedade, ampliando as possibilidades de identificação neste novo contexto. $\mathrm{O}$ foco do trabalho no modo psicossocial não é a doença, mas o usuário e seu entorno social ${ }^{(7)}$ e para atender as demandas implicadas nesta forma de cuidado é primordial a presença de uma equipe plural e comprometida com o trabalho.

A estrutura física foi referida em segundo lugar, entre os componentes da estrutura, como causa de incômodo aos trabalhadores de CAPS e SRT, assim como foi apontada por trabalhadores de CAPS de estado do centro-oeste brasileiro ser causa de descontentamento a inadequação do local de trabalho e precariedade do ambiente ${ }^{(8)}$.

No processo, as situações relacionadas à equipe apresentaram-se com maior relevância por incomodar os trabalhadores, corroborando afirmação de que a deterioração das relações de trabalho poderá interferir no fazer e na qualificação do trabalhador e também na construção de projetos profissionais, influenciando a saúde mental dos trabalhadores nele envolvidos $^{(9)}$. As relações de trabalho em serviços substitutivos de saúde mental são fundamentais dado que nestes a organização do trabalho é definida pelos acordos internos e pelas condições de execução do trabalho, e é dessa forma que cada equipe estabelece seu processo de trabalho ${ }^{(10)}$.

Outro aspecto do trabalho identificado em causar incomodo foi relativo à gestão, o que pode ser reflexo de dificuldades dos municípios em qualificar sua capacidade gestora, visto que, com a descentralização do Sistema Único de Saúde, as maiores atribuições de gestão das redes de serviços de saúde estão nos municípios $^{(11)}$. Também podemos ponderar que os modelos de gestão em saúde instituídos mostram-se inadequados e insuficientes para acolher serviços inovadores e complexos, como os que compõem a rede necessária ao modo psicossocial de cuidado ${ }^{(12)}$.

Entre as situações mais arroladas estão também aquelas que dizem respeito à rede de atenção e serviços, em consonância com estudo que identificou como uma das principais dificuldades no trabalho de CAPS a articulação entre serviços e outros equipamentos de saúde $^{(13)}$. Considera-se que são as equipes dos serviços que precisam responder pelos impasses decorrentes da 
inexistência de fluxos para recebimento e encaminhamento de usuários, dificultando ou inviabilizando o acompanhamento dos usuários fora dos serviços.

A atenção à crise no contexto psicossocial é entendida como resultante de vários fatores que ultrapassam o âmbito individual (biológico e psicológico) e compreendem também a família, os vizinhos, os amigos ou mesmo desconhecidos. E mesmo fazendo parte do quadro de possibilidades apresentadas no cotidiano dos serviços de SRT e CAPS, ainda é situação considerada problemática aos trabalhadores de serviços substitutivos ${ }^{(14)}$. No presente estudo está entre as quatro situações relacionadas ao processo mais referidas pelos trabalhadores em causar incômodo no trabalho.

Quanto ao perfil dos trabalhadores, os resultados mostram que, apesar das peculiaridades das duas modalidades de serviços estudadas - SRT e CAPS - os trabalhadores neles inseridos não manifestaram diferenças na percepção de situações que incomodam no trabalho. Ao analisar a escolaridade dos trabalhadores, a maior referência em enfrentar situações que incomodam no trabalho expressa pelos trabalhadores com curso superior e pós-graduação pode estar relacionada com maior expectativa daqueles com formação em curso superior quanto ao seu trabalho.

Os trabalhadores que estão nos serviços por tempo maior referem mais situações que incomodam, possivelmente por estarem expostos ao cotidiano do trabalho há mais tempo, terem vivências diferentes ao longo do período $\mathrm{e}$, consequentemente, mais elementos para analisar o seu trabalho. Em contrapartida, aqueles que se encontram no serviço por período menor podem não estar totalmente inseridos e não possuir sentimento de pertencimento ao contexto, dificultando a percepção do cotidiano do trabalho.

Quanto ao vínculo empregatício dos trabalhadores, podemos observar que as práticas de contratação através de estratégias alternativas aos concursos públicos e Consolidação das Leis Trabalhistas têm sido utilizadas para vincular o trabalhador ao serviço público de saúde como forma de atender a necessidade de expansão das contratações para ampliação da rede de serviços $^{(11)}$. Nesse cenário de flexibilização das relações trabalhistas, os vínculos podem ser estabelecidos de forma precária e acarretar prejuízo no estabelecimento de vínculo com usuários, de relações de confiança e no processo de cooperação entre os trabalhadores. E, consequentemente, colocá-los em uma situação de vulnerabilidade, indiferentes ou silenciosos diante de situações potenciais ao desagrado. A falta de relações duradouras e de representação sindical a que estão submentidos os trabalhadores com vínculos de trabalho precários contribuem para a alienação e dificultam a mobilização coletiva ${ }^{(15)}$.

\section{CONSIDERAÇÕES FINAIS}

A percepção dos trabalhadores acerca do seu trabalho mostrou que o processo, e em especial as relações constituídas no trabalho em equipe, são preponderantes em causar desagrado entre os trabalhadores. Isso reflete que o cuidado em liberdade aos indivíduos em sofrimento psíquico pode tensionar as relações intra equipe, assim como com gestores e demais serviços da rede de atenção à saúde e estas serem centralizadoras das dificuldades enfrentadas.

Considerando esse contexto, torna-se premente o investimento em políticas de formação que priorizem a capacitação para o cuidado em liberdade e o trabalho em equipes plurais, abertas aos profissionais de outras áreas de conhecimento, além da saúde. Também é necessário que os gestores estejam comprometidos com o modo psicossocial e capacitados para trabalhar de forma descentralizada, participativa e articulada com os demais segmentos da comunidade.

Por este ser um estudo quantitativo de corte transversal e ter utilizado questionários autoaplicáveis está sujeito à confiabilidade das informações prestadas, assim como ao estado emocional do participante no momento da pesquisa. Estudos longitudinais que pesquisem os trabalhadores em outros momentos de seu cotidiano fornecerão maiores informações para avaliação do trabalho no modo psicossocial.

\section{REFERÊNCIAS}

1. Amarante P. Loucos pela vida: a trajetória da reforma psiquiátrica no Brasil. Rio de Janeiro:Fiocruz; 2005.

2. Ministério da Saúde (BR). Secretaria de Atenção à Saúde. Saúde mental no SUS: os centros de atenção psicossocial. Brasília; 2004.

3. Ministério da Saúde (BR). Secretaria de Atenção à Saúde. Residências terapêuticas: o que são, para que servem. Brasília; 2004.

4. Milhomem MAGC, Oliveira AGB. O trabalho em equipe nos centros de atenção psicossocial - CAPS. Cogitare enferm. [Internet] 2007;12(1) [acesso em 14 ago 2012]. Disponível: http://ojs.c3sl.ufpr.br/ojs2/index. php/cogitare/article/view/8277/5786 
5. Universidade Federal de Pelotas. Faculdade de Enfermagem. Redes que reabilitam: avaliando experiências inovadoras de composição de redes de atenção psicossocial (REDESUL); coordenação Kantorski LP. Pelotas, 2011. 418p. Relatório final.

6. Donabedian A. Basic approaches to assessment: structure, process and outcome. In: Explorations in Quality Assessment and Monitoring, vol. 1. Ann Arbor: University of Michigan, Health Administration Press; 1980. p. 77-125.

7. Mielke FB, Kantorski LP, Olschowsky A, Jardim VMR. Características do cuidado em saúde mental em um CAPS na perspectiva dos profissionais. Trab. educ. saúde. [Internet] 2011;9(2) [acesso em 10 ago 2012]. Disponível:http://dx.doi.org/10.1590/S19817462011000200006

8. Rézio LA, Oliveira AGB. Equipes e condições de trabalho nos centros de atenção psicossocial em Mato Grosso. Esc. Anna Nery. [Internet]. 2010;14(2) [acesso 14 ago 2012]. Disponível: http://www.scielo.br/pdf/ean/ v14n2/18.pdf

9. Lancman S, Mângia EF, Gonçalves RMA, Jardim TA. Violência no trabalho e saúde mental em um serviço de pronto atendimento hospitalar. In: Assunção AA, Brito J, organizadores. Trabalhar na saúde: experiências cotidianas e desafios para a gestão do trabalho e do emprego. Rio de Janeiro: Fiocruz; 2011. p. 111-30.

10. Remminger T, Nardi HC. Modos de subjetivação dos trabalhadores de saúde mental em tempos de reforma psiquiátrica. Physis. [Internet] 2007;17(2) [acesso em 21 jul 2012]. Disponível: http://www. scielo.br/scielo.php?script $=$ sci_serial\&pid $=0103$ 7331\&lng=pt\&nrm=iso

11. Machado MH, Koster I. Emprego e trabalho em saúde no Brasil: as políticas de desprecarização do Sistema Único de Saúde. In: Assunção AA, Brito J, organizadores. Trabalhar na saúde: experiências cotidianas e desafios para a gestão do trabalho e do emprego. Rio de Janeiro: Fiocruz, 2011. p.195-213.

12. Silva MT, Lancman S, Alonso CMC. Conseqüências da intangibilidade na gestão dos novos serviços de saúde mental. Rev. Saúde Pública. [Internet] 2009;43(Suppl 1) [acesso em 19 jul 2012]. Disponível: http://dx.doi. org/10.1590/S0034-89102009000800007

13. Nascimento AF, Galvanese ATC. Avaliação da estrutura dos centros de atenção psicossocial do município de São
Paulo. Rev. Saúde Pública. [Internet]. 2009;43(Suppl 1) [acesso em 19 jul 2012]. Disponível: http://dx.doi. org/10.1590/S0034-89102009000800003

14. Zerbetto SE, Efigênio EB, Santos NLN, Martins SC. O trabalho em um Centro de Atenção Psicossocial: dificuldades e facilidades da equipe de enfermagem. Rev. Eletr. Enf. [Internet]. 2011;13(1) [acesso em 14 ago 2012]. Disponível: http://www.fen.ufg.br/revista/v13/n1/ v13nla11.htm.

15. Perissé NB, Alevato HMR. Impactos da terceirização na subjetividade do trabalhador [apresentação ao VIII Congresso Nacional de Excelência em Gestão; 8-9 jun 2012, Rio de Janeiro, Brasil].

Cogitare Enferm. 2012 Out/Dez; 17(4):697-702 